\title{
A Tracking Approach to Parcellation of the Cerebral Cortex
}

\author{
Chris Adamson ${ }^{1,2}$, Leigh Johnston ${ }^{1}$, Terrie Inder ${ }^{1}$, Sandra Rees ${ }^{4}$, Iven \\ Mareels $^{2}$, and Gary Egan ${ }^{1,3}$ \\ 1 Howard Florey Institute, University of Melbourne, Australia \\ Chris.Adamson@hfi.unimelb.edu.au \\ 2 Dept. of Electrical and Electronic Engineering, Univ. of Melbourne, Australia \\ 3 Centre For Neuroscience, University of Melbourne, Australia \\ 4 Department of Anatomy and Cell Biology, University of Melbourne, Australia
}

\begin{abstract}
The cerebral cortex is composed of regions with distinct laminar structure. Functional neuroimaging results are often reported with respect to these regions, usually by means of a brain "atlas". Motivated by the need for more precise atlases, and the lack of model-based approaches in prior work in the field, this paper introduces a novel approach to parcellating the cortex into regions of distinct laminar structure, based on the theory of target tracking. The cortical layers are modelled by hidden Markov models and are tracked to determine the Bayesian evidence of layer hypotheses. This model-based parcellation method, evaluated here on a set of histological images of the cortex, is extensible to 3-D images.
\end{abstract}

\section{Introduction}

The cerebral cortex is divided into specialised regions that are likely to be characterised by their laminar structure [see Figure 1(i)]. Many brain functions are localised to individual regions, and a major challenge of functional neuroimaging research involves the correlation of these regions to specific brain functions. Functional neuroimaging studies typically report results with respect to a structural atlas, the most popular in current studies being that of Brodmann [1] [see Figure 1(ii)]. However, the embryonic and post-natal formation of these regions is influenced by a combination of personal experience (environmental) and genetic influences, leading to unknown degrees of inter-subject variability in the overall cortical structure. Thus, individualised structural maps have applications in understanding normal human brain function as well as in the diagnosis and treatment of neurological and psychiatric diseases.

Inconsistencies arose in early postmortem atlases due to differences in experimental conditions and subjective layering schemes [2]. More recently, analytical techniques [2-4] have focused on automatic, objective identification of homogeneous regions of cortical structure in digitised images of postmortem brain sections. However, these classification methods all use inferential statistics without directly modelling the layering structure. Therefore, these schemes are sensitive to relative layer depth changes, which occur due to factors such as curvature of the cortex and the obliqueness of histological slice cuts, and are difficult to account for. 


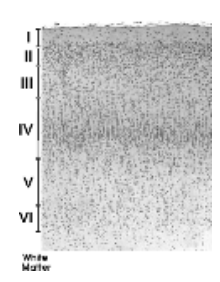

(i)

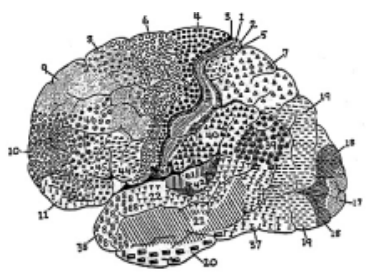

(ii)

Fig. 1. (i) High-magnification image of haematoxylin and eosin (H \& E) stained histological section of primary visual cortex from a baboon brain, with Roman numerals denoting the layering structure. (ii) Brodmann's cytoarchitectonic atlas for the human cortex, lateral view, from [1].

The limited number of parcellation schemes that directly model the laminar structure and are extensible to a 3D setting motivate this paper [5]. We propose a novel target tracking-based method that models the cortical layers using hidden Markov models and computes Bayesian evidence of layering to determine parcellation. Our method is grounded in the work of Xie and Evans [6], who track dominant frequencies in noisy time-varying signals using hidden Markov models. We present this paper as work that forms the basis for a method that is able to parcellate the cortex in a 3D Magnetic Resonance Image (MRI) of a living subject, as visualised in a number of recent experimental papers [7-9].

\section{Method}

\subsection{Image Acquisition and Sampling}

Coronal sections of a baboon cortex were stained with haematoxylin and eosin $(\mathrm{H} \& \mathrm{E})$ and the histological images, of resolution $14 \mu \mathrm{m} \times 14 \mu \mathrm{m}$, were captured using a Nikon LS-2000 slide scanner [see Figure 2(i)].

We sample the cortex using a procedure that is directly extensible to 3-D, based on the method of Jones et al. [10, which solves Laplace's equation $\left(\nabla^{2} \Psi=\right.$ $0)$. Here $\Psi$ is a potential function defined subject to the Dirichlet boundary conditions on the White Matter/Grey Matter $(\mathrm{WM} / \mathrm{GM})$ boundary $(\Psi(\mathrm{WM} / \mathrm{GM})=0)$ and the Grey Matter/Cerebral Spinal Fluid (pial) boundary $(\Psi($ pial $)=1)$. Finite differences are used to determine a solution to Laplace's equation. The tangent field to this solution can be traced to define lines that span the cortex.

The raw intensity profiles resulting from this method are extremely noisy due to the high degree of texture variation in the images caused by the histological staining processes. For this reason the data is mean filtered prior to parcellation, thus smoothing the local image intensity variations. Choice of the optimal filter size is discussed in Section 3 .

The inherent ordering in the set of sampled profiles implies a sequence of vectors, equivalently a matrix. When visualised as a $2 \mathrm{D}$ image, this ap- 


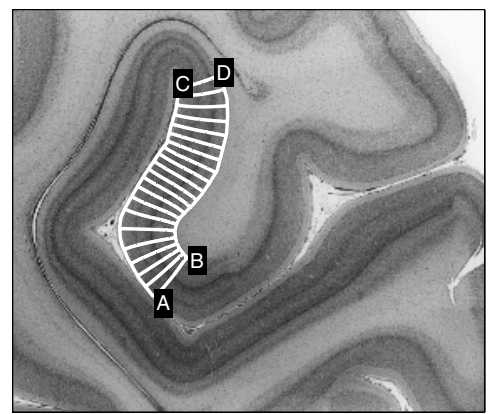

(i)

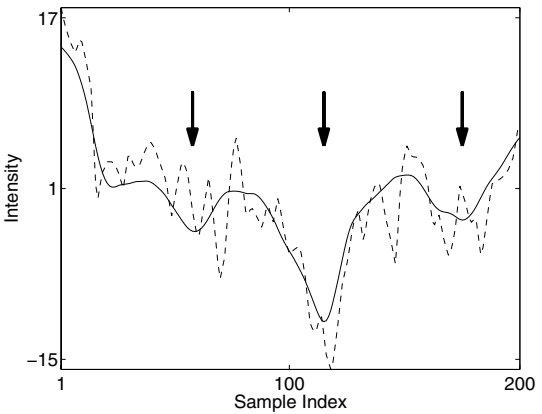

(ii)

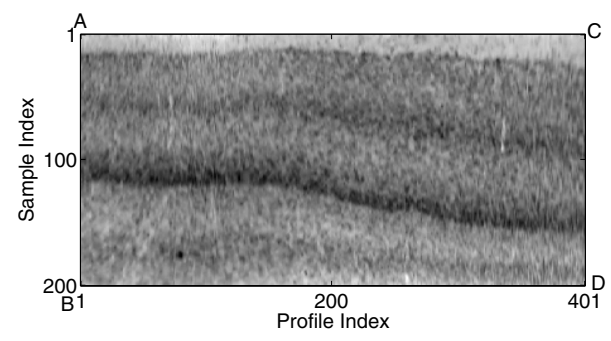

(iii)

Fig. 2. (i) Histological slice of baboon cortex, with a section of the WM/GM and pial boundary contours and a sub-sample of the lines overlaid. (ii) Intensity profile 100 (dashed line $=$ original, solid line $=$ mean-filtered), arrows indicate centres of troughs. (iii) "Straightened" representation of section in Fig.(i).

pears to be a "straightened" representation of the sampled section of the cortex [see Figure 2(iii)]. We denote by $\mathbf{Y}_{1}^{T}=\left[\mathbf{y}_{1}, \ldots, \mathbf{y}_{T}\right]$ the sequence of $T$ filtered line profiles, where each profile is a $K$-vector of image intensity values, $\mathbf{y}_{t}=\left[y_{1, t}, \ldots, y_{K, t}\right]^{\prime}, y_{k, t} \in \mathbb{R}$.

\subsection{Tracking-Based Parcellation of the Cortex}

The strongest visual cue to the identification of laminar structure in the cortex is the presence of dark bands [see Figure 2(iii)]. Each distinct region in the cortex as observed in a typical histology image [see Figure 2(i)] occupies an extensive area. Thus, the dark bands that constitute these regions extend across hundreds of sampled profiles. However, the boundaries between bands in individual profiles are not always sharp and in some cases small, transient bands exist. Therefore, while a visual parcellation of the images is possible, constructing an algorithm that is able to mimic this level of non-local information processing is a difficult task.

After appropriate filtering, the dark bands manifest as troughs in the intensity profiles [see Figure 2(ii)]. The bottom of the troughs in a profile are the estimated centres of the dark bands that exist in that profile. 
We have used a target tracking paradigm, since that cortical layers have predictable local dynamics well-suited to Markov modelling [11. That is, by considering the profile index to be a temporal variable, the probabilistic centroid of a dark band is a "target" that we wish to track, taking into account its "movement" up and down through "time".

The Markov chain state is used to denote the position of a track in the image, with the movement up and down of the bands captured by suitable choice of the transition probability matrix. The profiles are the noisy observations of these underlying states (band positions).

Introducing notation for these concepts, we define a track to be a sequence of pixel indices delineating the probabilistic centre of a dark band in the image that starts at position, $\left(k_{0}, t_{0}\right)$, and exists for a length of $n$ pixels:

$$
\mathbf{d}^{\left(k_{0}, t_{0}, n\right)}=\left[d_{t_{0}}^{\left(k_{0}, t_{0}, n\right)}=k_{0}, d_{t_{0}+1}^{\left(k_{0}, t_{0}, n\right)}, \ldots, d_{t_{0}+n-1}^{\left(k_{0}, t_{0}, n\right)}\right]
$$

where $d_{t}^{\left(k_{0}, t_{0}, n\right)} \in\{1, \ldots, K\}, \forall t=t_{0}+1, \ldots, t_{0}+n-1$. Let $\Omega=\left\{\left(k_{0}, t_{0}, n\right)\right\}$ denote the set of all track parameter 3 -tuples.

We assume first-order Markov dynamics for the tracks, whereby the position of the track in profile $t$ depends only on the position of the track in profile $t-1$. Thus we define a transition probability matrix, $A$, with elements,

$$
a(j, k)=p\left(d_{t}^{\left(k_{0}, t_{0}, n\right)}=k \mid d_{t-1}^{\left(k_{0}, t_{0}, n\right)}=j\right)
$$

The transition matrix $A$ is constructed to be diagonally dominant, reflecting the biological constraint that limited local "movement" is more probable than drastic "movement."

The likelihood of a profile given a track state is constructed by assuming firstly only local dependence of the data, within a window size $m$,

$$
p\left(\mathbf{y}_{t} \mid d_{t}^{\left(k_{0}, t_{0}, n\right)}=k\right)=p\left(\left[y_{k-m, t}, \ldots, y_{k, t}, \ldots, y_{k+m, t}\right] \mid d_{t}^{\left(k_{0}, t_{0}, n\right)}=k\right)
$$

and secondly, that a quadratic fitted to the data $\left[y_{k-m, t}, \ldots, y_{k, t}, \ldots, y_{k+m, t}\right]$ will have it's turning point, $(\hat{k}, \hat{t})$, close to pixel $(k, t)$ if this pixel is at the centre of a trough, and further away when the pixel is not at the centre of a trough. We therefore construct the likelihood function as

$$
\begin{aligned}
p\left(\mathbf{y} \mid d_{t}^{\left(k_{0}, t_{0}, n\right)}=k\right) & =p\left(\rho\|(k, t)-(\hat{k}, \hat{t})\| \mid d_{t}^{\left(k_{0}, t_{0}, n\right)}=k\right) \\
\rho\|(k, t)-(\hat{k}, \hat{t})\| & \sim N(0,1)
\end{aligned}
$$

where $\rho \in \mathbb{R}$ scales distance measure to make unit variance applicable.

Having thus formulated the hidden Markov model of the tracks, we describe the algorithm for parcellation based on this model. We calculate the evidence for the hypothesis, $H_{k, t}$, that a pixel $(k, t)$ is situated at the probabilistic centre of a dark band. Evidence is measured by the posterior probability of the hypothesis given the data:

$$
p\left(H_{k, t} \mid \mathbf{Y}_{1}^{T}\right), \quad \forall k=1, \ldots, K, \quad t=1, \ldots, T
$$


A pixel is situated at the probabilistic centre of a dark band if and only if a track, as defined in (1), passes through that point. That is, that there exists some $\left(k_{0}, t_{0}, n\right)$ for which $d_{t}^{\left(k_{0}, t_{0}, n\right)}=k$. Therefore by the law of total probability, (6) can be expressed in terms of a sum of posterior probabilities over all possible tracks:

$$
p\left(H_{k, t} \mid \mathbf{Y}_{1}^{T}\right)=\sum_{\left\{k_{0}, t_{0}, n\right\} \in \Omega} p\left(d_{t}^{\left(k_{0}, t_{0}, n\right)}=k \mid \mathbf{Y}_{1}^{T}\right), \forall k=1, \ldots, K, t=1, \ldots, T
$$

The posterior probability of pixel $(k, t)$ belonging to track $\mathbf{d}^{\left(k_{0}, t_{0}, n\right)}$ is rewritten using Bayes' rule as

$$
p\left(d_{t}^{\left(k_{0}, t_{0}, n\right)}=k \mid \mathbf{Y}_{1}^{T}\right)=\frac{p\left(\mathbf{Y}_{1}^{T} \mid d_{t}^{\left(k_{0}, t_{0}, n\right)}=k\right) p\left(d_{t}^{\left(k_{0}, t_{0}, n\right)}=k\right)}{p\left(\mathbf{Y}_{1}^{T}\right)}
$$

The prior in (8) is considered to contain a local and a global term:

$$
p\left(d_{t}^{\left(k_{0}, t_{0}, n\right)}=k\right)=p_{l o c}\left(d_{t}^{\left(k_{0}, t_{0}, n\right)}=k\right) p_{\text {glob }}\left(d_{t}^{\left(k_{0}, t_{0}, n\right)}=k\right)
$$

The local prior is the first-order Markov assumption discussed above. The global prior is a weighting term on the length of the track, such that longer tracks are more probable than shorter ones. This local/global factorisation of the prior term is used as a tool in image processing methods that attempt to capture both short and long range properties of an object of interest 12 .

We make a conditional independence assumption on the image data; profiles that are not between $t_{0}$ and $\left(t_{0}+n-1\right)$ are independent of the track state $d_{t}^{\left(k_{0}, t_{0}, n\right)}$. Let $\mathbf{Y}_{t_{0}}^{t_{0}+n-1}$ be the sequence of profiles between $t_{0}$ and $t_{0}+n-1$. Then, by cancelling independent data in numerator and denominator, and by reverse application of Bayes' rule, (8) becomes

$$
p\left(d_{t}^{\left(k_{0}, t_{0}, n\right)}=k \mid \mathbf{Y}_{t_{0}}^{t_{0}+n-1}\right) \propto p_{l o c}\left(d_{t}^{\left(k_{0}, t_{0}, n\right)}=k \mid \mathbf{Y}_{t_{0}}^{t_{0}+n-1}\right) p_{g l o b}\left(d_{t}^{\left(k_{0}, t_{0}, n\right)}=k\right)
$$

Here, $p_{l o c}\left(d_{t}^{\left(k_{0}, t_{0}, n\right)}=k \mid \mathbf{Y}_{t_{0}}^{t_{0}+n-1}\right)$ is a state occupancy probability, computed by the polynomial-time hidden Markov model (HMM) smoother, commonly known as the forward-backward algorithm [11]. The HMM smoother computes the state occupancy probability via

$$
p_{l o c}\left(d_{t}^{\left(k_{0}, t_{0}, n\right)}=k \mid \mathbf{Y}_{t_{0}}^{t_{0}+n-1}\right)=\alpha_{t}(k) \beta_{t}(k) /\left(\sum_{k=1}^{K} \alpha_{t}(k) \beta_{t}(k)\right)
$$

where $\alpha_{t}(k)$ and $\beta_{t}(k)$ are determined in forward and backward sweeps through the data respectively (see 11] for details).

The initial condition that the track starts in pixel $\left(k_{0}, t_{0}\right)$ is reflected by the initialisation of the forward variable:

$$
\alpha_{t_{0}}(k)= \begin{cases}1, & k=k_{0} \\ 0, & \text { otherwise }\end{cases}
$$

The backward variable is initialised to reflect that the track can end in any state:

$$
\beta_{t_{0}+n-1}(k)=1, \forall k=1, \ldots, K
$$


The computational requirement in running an HMM smoother for all possible track parameters, $\left(k_{0}, t_{0}, n\right)$, to calculate the posterior probability of the evidence (7) at each pixel is prohibitive. Instead, we limit the size of the parameter space, $\Omega$, by considering only a set of points that are highly likely to be the start of tracks, as measured by a large change in likelihood between two horizontallyneighbouring pixels.

Having computed the posterior probability (7) for all pixels in the image, we sweep the image left-to-right and classify regions according to the appearance and disappearance of tracks.

\section{Results}

The results of applying the proposed method to three example histological images acquired as described in Section 2 are shown in Figures 3 . The size of the mean filter was chosen to be $10 \times 100$ pixels, optimising the trade-off between

(i)

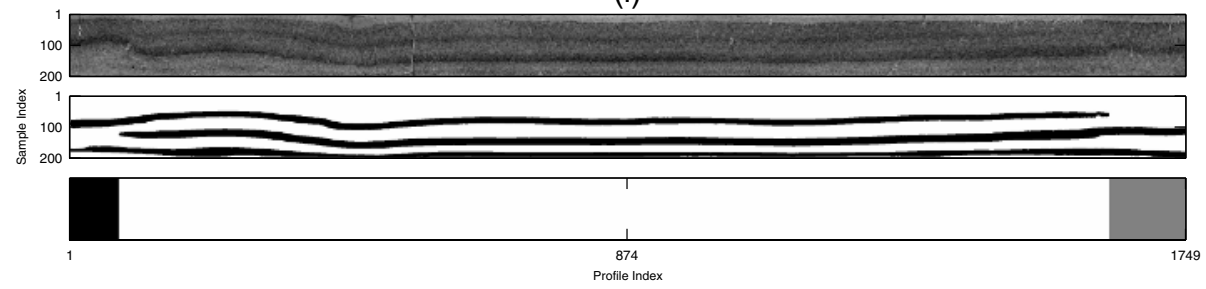

(ii)

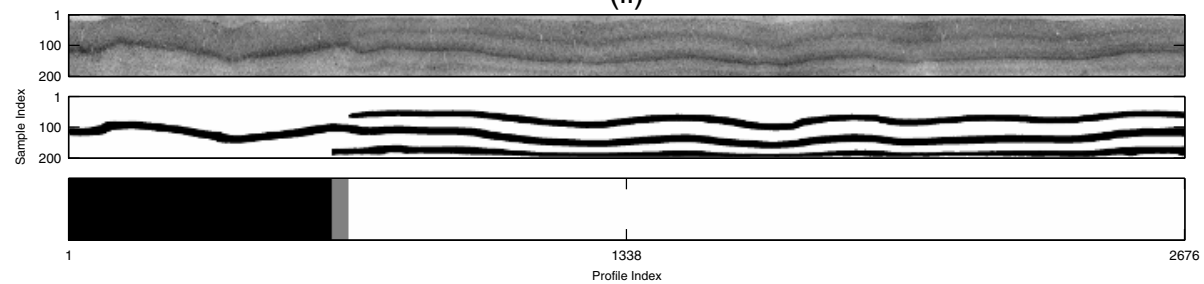

(iii)

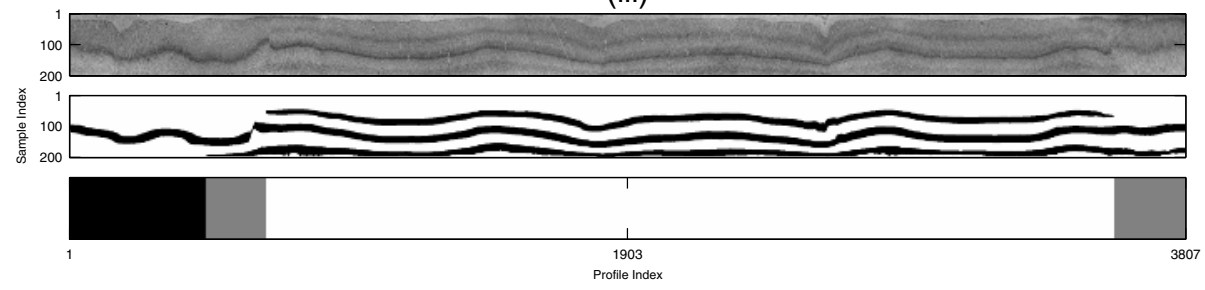

Fig. 3. (i)-(iii) Three histological sections of baboon cortex from different parts of a single brain. In each, Top: "straightened" representation of profiles, Middle: posterior probability map, dark indicates higher probability, Bottom: parcellation of line profiles; note that there is no correspondence between regions with the same colour in different figures. 
unwanted image blur and noise texture smoothing. The likelihood normalising factor was set to $\rho=200$, while the global prior was chosen to be a $N(T, 500)$ distribution, where $T$ is the width of the image, and $\sigma^{2}=500$ was chosen to appropriately weight a range of longer tracks, i.e. those tracks more likely to within a dark band. The tuning parameters, $\rho$ and $\sigma^{2}$, while somewhat arbitrary, are analogous to tuning parameters in target tracking schemes which are in general also arbitrarily chosen.

As is evidenced in Figure 3, the parcellation into regions of distinct laminar structure has been accomplished by the proposed method. The small transition region (grey region between black and white) in Figure 3(ii) is caused by the extension of the bottom dark band past the termination of the topmost dark band. The transition region in Figure 3(iii) is caused by an erroneous weak trough attaching to a stronger dark trough. We are currently investigating likelihood models that suppress weak troughs more effectively.

\section{Conclusions}

The probabilistic, model-based method proposed in this paper improves on previous parcellation schemes, both in it's applicability to 3D data, and it's modelling of the layer structure known to be present in the data. In this latter regard, our algorithm makes use of the prior information known about the data, i.e. direct identification of the biological structure underlying the problem, and is therefore more robust and generalisable than methods based solely on inferential statistical measures. We have demonstrated that our method produces a parcellation that contains contiguous regions that correspond to discernible regions of homogeneous laminar structure.

The target tracking paradigm we employ models the probabilistic centres of the dark bands in the cortex by hidden Markov models and tracks their position through the data. A notion of causality is imposed on the data by considering the horizontal axis to be a temporal variable, which is possible due to the sequential organisation of the profiles.

Parcellation in 3D is considerably more difficult, namely that the $1 \mathrm{D}$ linear profile arrangement is replaced by a $2 \mathrm{D}$ graphical organisation. This $2 \mathrm{D}$ arrangement has no notion of causality, which is exploited in a target tracking paradigm. However, certain fundamental aspects of the algorithm, such as robustness to band depth changes and image artifacts will form the basis of a 3D parcellation algorithm, which is the focus of current work.

\section{References}

1. Garey, L.J.: Brodmann's Localisation in the Cerebral Cortex. Smith-Gordon, London (1994)

2. Schleicher, A., Amunts, K., Geyer, S., Morosan, P., Zilles, K.: ObserverIndependent Method for Microstructural Parcellation of Cerebral Cortex: A Quantitative Approach to Cytoarchitectonics. NeuroImage 9 (1999) 165 - 177 
3. Annese, J., Pitiot, A., Dinov, I.D., Toga, A.W.: A myelo-architectonic method for the structural classification of cortical areas. NeuroImage 21 (2004) $15-26$

4. Adamson, C., Davies, R., Inder, T., Rees, S., Mareels, I., Egan, G.: Markov random field-based parcellation of the cerebral cortex: Application to histology images. In: ISSNIP 2004. Volume 1. (2004) 559 - 564

5. Kruggel, F., Brückner, M.K., Arendt, T., Wiggins, C.J., von Cramon, D.Y.: Analyzing the Neocortical Fine-Structure. Medical Image Analysis 7 (2003) 251 264

6. Xie, X., Evans, R.J.: Multiple target tracking and multiple frequency line tracking using hidden Markov models. IEEE Transactions on Signal Processing 39 (1991) $2659-2676$

7. Walters, N., Egan, G., Kril, J., Kean, M., Waley, P., Jenkinson, M., Watson, J.: In vivo Identification of Human Cortical Areas using High-Resolution MRI: An Approach to Cerebral Structure-Function Correlation. Proceedings of the National Academy of Sciences 100 (2003) 2981 - 2986

8. Barbier, E.L., Marrett, S., Danek, A., Vortmeyer, A., van Gelderen, P., Duyn, J., Bandettini, P., Grafman, J., Koretsky, A.P.: Imaging Cortical Anatomy by HighResolution MR at 3.0T: Detection of the Stripe of Gennari in Visual Area 17. Magnetic Resonance in Medicine 48 (2002) 735 - 738

9. Bridge, H., Clare, S., Jenkinson, M., Jezzard, P., Parker, A.J., Matthews, P.M.: Independent anatomical and functional measures of the V1/V2 boundary in human visual cortex. Journal of Vision 5 (2005) $93-102$

10. Jones, S.E., Buchbinder, B.R., Aharon, I.: Three-Dimensional Mapping of Cortical Thickness Using Laplace's Equation. Human Brain Mapping 11 (2002) 12 - 32

11. Rabiner, L.R.: A tutorial on hidden Markov models and selected applications in speech recognition. Proceedings of the IEEE 77 (1989) $257-286$

12. Elder, J.H., Krupnik, A., Johnston, L.A.: Contour Grouping with Prior Models. IEEE Transactions on Pattern Analysis and Machine Intelligence 25 (2003) $661-674$ 